\title{
WELL-POSEDNESS AND REGULARITY OF HYPERBOLIC BOUNDARY CONTROL SYSTEMS ON A ONE-DIMENSIONAL SPATIAL DOMAIN*
}

\author{
Hans Zwart $^{1}$, Yann Le Gorrec ${ }^{2}$, Bernhard Maschke $^{3}$ and Javier Villegas ${ }^{4}$
}

\begin{abstract}
We study a class of hyperbolic partial differential equations on a one dimensional spatial domain with control and observation at the boundary. Using the idea of feedback we show these systems are well-posed in the sense of Weiss and Salamon if and only if the state operator generates a $C_{0}$-semigroup. Furthermore, we show that the corresponding transfer function is regular, i.e., has a limit for $s$ going to infinity.
\end{abstract}

Mathematics Subject Classification. 93C20, 35L40, 35F15, 37Kxx.

Received April 3rd, 2008. Revised April 1st, 2009.

Published online August 25, 2009.

\section{INTRODUCTION}

Consider the abstract linear differential equation

$$
\begin{aligned}
& \dot{x}(t)=A x(t)+B u(t), \quad x(0)=x_{0} \\
& y(t)=C x(t)+D u(t),
\end{aligned}
$$

where $x$ is assumed to take values in the Hilbert space $X, u$ in the Hilbert space $U$, and $y$ in the Hilbert space $Y$. The operators $A, B, C$, and $D$ are linear operators. Under the standard assumption that $A$ generates a $C_{0}$-semigroup, we know that the homogeneous equation, i.e., $u \equiv 0$ in (1.1) has a unique (weak) solution. Assuming that $B, C$, and $D$ are bounded operators, there exists a solution of (1.1)-(1.2) for every $u \in L^{2}\left(\left(0, t_{f}\right) ; U\right)$. Existence of solutions for an arbitrary initial condition $x_{0} \in X$ and input $u \in L^{2}\left(\left(0, t_{f}\right) ; U\right)$, such that $y \in L^{2}\left(\left(0, t_{f}\right) ; Y\right)$ is called well-posedness, see e.g. [23,26]. Hence if $B, C$, and $D$ are bounded linear operators,

Keywords and phrases. Infinite-dimensional systems, hyperbolic boundary control systems, $C_{0}$-semigroup, well-posedness, regularity.

* The contributions of Y. Le Gorrec and B. Maschke have been done within the context of the European sponsored project GeoPlex with reference code IST-2001-34166 and French NRA sponsored project RECIPROC with reference code ANR-06-JCJC0011. Further information is available at http://www.geoplex.cc and http://www.agence-nationale-recherche.fr/Intl.

${ }^{1}$ Department of Applied Mathematics, University of Twente, 7500 AE Enschede, The Netherlands. h.j.zwart@math.utwente.nl

2 FEMTO-ST AS2M, 24 rue Alain Savary, 25000 Besançon, France. Yann.Le.Gorrec@ens2m.fr

${ }^{3}$ LAGEP, CNRS UMR 5007, CPE Lyon - Bâtiment 308 G, Université Lyon-1, Université de Lyon, 43 bd. du 11 Novembre 1918, 69622 Villeurbanne Cedex, France. maschke@lagep.univ-lyon1.fr

${ }^{4}$ AVL Powertrain UK, Langdale House, Sable Way, Southfields Business Park, Basildon, SS15 6SR, UK.

Javier.Villegas@avl.com 
then the system (1.1)-(1.2) is well-posed if and only if $A$ is the infinitesimal generator of a $C_{0}$-semigroup. For more details on this class of systems we refer to Chapters 2 and 3 of [2].

As it is known for a very long time not all linear partial differential equations (p.d.e.'s) can be written into the format (1.1)-(1.2) with a bounded $B$ and $C$. Consider for instance, the delay line on the interval $[0,1]$ which is modelled as

$$
\begin{aligned}
\frac{\partial w}{\partial t}(t, z) & =\frac{\partial w}{\partial z}(t, z), \quad w(0, z)=w_{0}(z), \quad z \in[0,1] \\
u(t) & =w(t, 1) \\
y(t) & =w(t, 0)
\end{aligned}
$$

Without going into too much detail, it is clear that the mapping $f \mapsto f(0)$ is not a bounded mapping on the state space $X=L^{2}(0,1)$ to the output space $\mathbb{C}$. Hence $C$ is not a bounded operator. Similarly, $B$ is not a bounded operator from $\mathbb{C}$ to $X$ either. It is not hard to see that for any $w_{0} \in X$ and $u \in L^{2}(0, \infty)$, the solution of (1.3)-(1.5) is given by $w(t, z)=f(t+z)$ and $y(t)=f(t)$, where $f(\xi)=w_{0}(\xi), \xi \in[0,1]$ and $f(\xi+1)=u(\xi), \xi>0$. So if $w_{0} \in X=L^{2}(0,1)$, and $u \in L^{2}(0, \infty)$, then $f \in L^{2}(0, \infty)$ and so $w(t, \cdot) \in X$ for all $t>0$, and $y \in L^{2}(0, \infty)$. Hence this system is well-posed, without having bounded $B$ and $C$ operators.

Over the last decades there has been a growing interest in well-posedness of p.d.e.'s with control and observation on the boundary. The main books on this subject are the books by Lasiecka and Triggiani [12,13] and by Tucsnak and Weiss [24]. The references in these books together with Section 5.8 of Staffans [23] give a good starting point for the interested reader. Additionally, we discuss a few of the recent papers which treat physically motivated (hyperbolic) p.d.e.'s. In [5] well-posedness is investigated for the Schrödinger equation. The Euler Bernoulli beam is treated in [6,8], whereas well-posedness of the wave equation is the subject of $[7,9,10]$. These papers and also many examples in the books $[12,13,24]$ focus on well-posedness of p.d.e.'s on a two or three dimensional spatial domain. We treat systems on a one-dimensional spatial domain. In contrast to the higher dimensional situation, for the one-dimensional case a single proof covers the well-posedness for the whole class. Our class of p.d.e.'s stems from a Hamiltonian formulation of physical models, called boundary portHamiltonian systems [15,25], defined on a one-dimensional spatial domain. This class includes models of the one-dimensional (undamped) wave equation and Timoshenko beam. It also includes networks of elementary systems such as vibrating strings or delay lines, see $[3,4,16]$. The aim of this paper is to show that for this class we have a similar theorem as for the class of systems with bounded $B$ and $C$, see (1.1) and (1.2). Namely, it is well-posed if and only if the homogeneous equation, i.e., $u \equiv 0$ generates a $C_{0}$-semigroup.

The set-up of the proof is relatively simple. First we consider a system that consists out of a collection of delay lines, e.g. (1.3)-(1.5). If these delay lines are non-connected, then it is very easy to show well-posedness. If they are connected, then we regard this connection as a feedback of the non-connected situation, see Figure 1 on p. 1085. Using a result by Weiss [26], we know that this connected case will lead to a well-posed system, provided the feedback gives a bounded closed loop transfer function. Hence we study the closed loop transfer function. For the considered class of systems we show that if the feedback connection does not give a bounded transfer function, then the homogeneous system does not generate a $C_{0}$-semigroup.

The general case is shown by performing a state basis transformation such that the system becomes a set of simple delay lines. This is rather standard by using the characteristic or the Riemann coordinates for our p.d.e. The idea of treating one-dimensional (hyperbolic) p.d.e.'s by this basis transformation has been used by Phillips [20] and Russell [21] fifty and thirty years ago, respectively. Phillips assumes that the semigroup is contractive. In [21] this is not assumed, but there the author considers non-mixed boundary conditions. On p. 649, Russell indicates why certain boundary conditions do not lead to a solution of the p.d.e. In the proof of Theorem 3.3 we give a more general argument. From Theorem 3.1 by Russell we see that if the homogeneous p.d.e. has an unique solution, then the same holds for the non-homogeneous one. However, well-posedness implies more, it also implies that the mapping from the input to the output is bounded, which for hyperbolic systems on a one-dimensional spatial domain can be non-trivial, see e.g. [27]. The reason why for our systems the proof is relatively easy lies in the fact that we are looking at a special class of hyperbolic p.d.e.'s, see (2.3), and use its 
structure in order to formulate the boundary control and observations using well-adapted (physically motivated) variables, see Section 5. In example 2.11 of [1] one may find an example showing that these variables leads to well-posed systems, whereas another choice does not. However, in that paper no underlying reason was given. Finally, we remark that our proof easily carries over to inputs and outputs in $L^{q}$, see Section 7 . A preliminary version of our results has been published in [29].

\section{Class of P.D.E.'S AND MAIN RESUlt}

For the sake of simplicity, we use the elementary hyperbolic p.d.e., i.e. the wave equation, as illustrative example

$$
\frac{\partial^{2} w}{\partial t^{2}}(t, z)=c^{2} \frac{\partial^{2} w}{\partial z^{2}}(t, z)
$$

We consider this as the model of a uniform vibrating string. In this case the variable $w(t, z)$ denotes the position with respect to equilibrium of the string at position $z$ and the constant $c$ denotes the propagation speed (celerity) $c=\sqrt{\frac{T}{\rho}}$ with $T$ Young's modulus and $\rho$ the mass density. However, the physical formulation of the model of the string is given as a set of coupled conservation laws and induces the following choice of the state variables: the momentum $x_{1}(t, z)=\rho \frac{\partial w}{\partial t}(t, z)$ and the elastic strain $x_{2}(t, z)=\frac{\partial w}{\partial z}(t, z)[18,22,25]$. It may be noticed that this formulation is more general than (2.1) as it remains valid in the case of a non-uniform string when Young's modulus $T$ and the mass density $\rho$ depend on $z$. In these variables the model of the vibrating string is written as follows:

$$
\begin{aligned}
\frac{\partial}{\partial t}\left(\begin{array}{c}
x_{1} \\
x_{2}
\end{array}\right)(t, z) & =\left(\begin{array}{ll}
0 & 1 \\
1 & 0
\end{array}\right) \frac{\partial}{\partial z}\left(\begin{array}{c}
\frac{1}{\rho} x_{1} \\
T x_{2}
\end{array}\right)(t, z) \\
& =\left(\begin{array}{ll}
0 & 1 \\
1 & 0
\end{array}\right) \frac{\partial}{\partial z}\left[\left(\begin{array}{cc}
\frac{1}{\rho} & 0 \\
0 & T
\end{array}\right)\left(\begin{array}{l}
x_{1} \\
x_{2}
\end{array}\right)\right](t, z) .
\end{aligned}
$$

We have given two examples, the first one corresponding to the delay line (1.3) consisting in one conservation law and the second one (2.2), the vibrating string, consisting in two coupled conservation laws. More examples of coupled systems of conservation laws may be found of hyperbolic type [17,18,25] but also of parabolic type [14,25].

In this paper we consider a general class of conservation laws with flux variables depending linearly on the state and linear source term:

$$
\frac{\partial x}{\partial t}(t, z)=P_{1} \frac{\partial}{\partial z}(\mathcal{L} x)(t, z)+P_{0}(z) x(t, z), \quad z \in[a, b]
$$

where $x$ is a function taking values in $\mathbb{R}^{n}, \mathcal{L}$ is a multiplication operator satisfying $0<m I \leq \mathcal{L}(z) \leq M I$, $z \in[a, b]$, for some positive constants $m$ and $M, P_{1}$ is a constant (real), invertible, matrix satisfying $P_{1}^{T}=P_{1}$ and $P_{0}$ is some matrix valued function.

With the operator $\mathcal{L}$ we introduce the Hilbert space $X$ as being the function space $L^{2}\left((a, b) ; \mathbb{R}^{n}\right)$ with the inner product

$$
\langle f, g\rangle_{\mathcal{L}}=\int_{a}^{b} f(z)^{*} \mathcal{L}(z) g(z) \mathrm{d} z .
$$

The norm associated to this inner product, denoted by $\|x\|_{\mathcal{L}}$, has, in the case of physical models, the meaning of energy. The operator $\mathcal{L}$ may be interpreted as the variational derivative of the energy $\mathcal{L} x=\frac{1}{2} \frac{\delta\|x\|_{\mathcal{L}}^{2}}{\delta x}$. Note that since $m I \leq \mathcal{L}(z) \leq M I$, this new norm is equivalent to the standard $L^{2}$-norm. 
Assume for a moment that $P_{0}$ is zero, and that we have a classical solution of the p.d.e. (2.3), then

$$
\begin{aligned}
\frac{\mathrm{d}}{\mathrm{d} t}\|x(t)\|_{\mathcal{L}}^{2}= & \left\langle\frac{\partial x}{\partial t}, x\right\rangle_{\mathcal{L}}+\left\langle x, \frac{\partial x}{\partial t}\right\rangle_{\mathcal{L}} \\
= & \left\langle P_{1} \frac{\partial}{\partial z}(\mathcal{L} x), x\right\rangle_{\mathcal{L}}+\left\langle x, P_{1} \frac{\partial}{\partial z}(\mathcal{L} x)\right\rangle_{\mathcal{L}} \\
= & \int_{a}^{b}\left(P_{1} \frac{\partial}{\partial z}(\mathcal{L} x)(t, z)\right)^{*} \mathcal{L}(z) x(z) \mathrm{d} z \\
& +\int_{a}^{b} x(z)^{*} \mathcal{L}(z) P_{1} \frac{\partial}{\partial z}(\mathcal{L} x)(t, z) \mathrm{d} z \\
= & \int_{a}^{b} \frac{\partial}{\partial z}\left[(\mathcal{L} x)^{*}(t, z) P_{1}(\mathcal{L} x)(t, z)\right] \mathrm{d} z \\
= & {\left[(\mathcal{L} x)^{*}(t, z) P_{1}(\mathcal{L} x)(t, z)\right]_{a}^{b}, }
\end{aligned}
$$

where we have used the symmetry of $P_{1}$ and $\mathcal{L}(z)$. Hence by putting enough boundary conditions to zero, this last expression becomes zero, and the energy remains constant along solutions. This reflects the hyperbolic nature of (2.3). From the calculation above, we can clearly see the advantage of writing a p.d.e. in the form (2.3). The symmetry of $P_{1}$ tells that the system is hyperbolic in nature, and the $\mathcal{L}$ tells us which energy function should be used. In many models the $P_{1}$ only contains ones and zeros, telling how different physical domains are coupled, and all the physical parameters are in $\mathcal{L}[14,17,18,25]$. One may also see $P_{1}$ as the matrix obtained by putting all physical constants to one. Standard p.d.e. theory gives that we do not have a unique solution of (2.3) if we do not impose boundary conditions. If we set all $2 n$ boundary conditions to zero, then we only have the zero solution, and so we cannot choose a non-zero initial condition. It is well-known that we must impose $n$ boundary conditions, if we want to have existence and uniqueness of solutions. In [20], Section 1.5, all boundary conditions are characterized for which we have that the energy stays constant along solutions. For a formulation of this result in the language of this paper, we refer to [15].

We decompose the set of $n$ boundary conditions into two sets, the first one contains those boundary conditions that are set to zero, whereas the other class contains the boundary conditions which are free to choose, i.e., the inputs. More precisely, we shall complete the p.d.e. (2.3) with a set of boundary conditions defined in terms of the variational derivative of the energy, $\mathcal{L} x$, and not the state variable $x$, as follows:

$$
\begin{aligned}
\frac{\partial x}{\partial t}(t, z) & =P_{1} \frac{\partial}{\partial z}(\mathcal{L} x)(t, z)+P_{0}(z) x(t, z), \quad x(0, z)=x_{0}(z) \\
0 & =M_{11}(\mathcal{L} x)(t, b)+M_{12}(\mathcal{L} x)(t, a) \\
u(t) & =M_{21}(\mathcal{L} x)(t, b)+M_{22}(\mathcal{L} x)(t, a)
\end{aligned}
$$

where $\left[\begin{array}{ll}M_{11} & M_{12} \\ M_{21} & M_{22}\end{array}\right]$ is a $n \times 2 n$ matrix with rank $n$. The maximality of the rank implies that $(2.7)$ and (2.8) are not conflicting. Furthermore, we assume that we observe the system through its boundary in terms of the variables $\mathcal{L} x$ :

$$
y(t)=C_{1}(\mathcal{L} x)(t, b)+C_{2}(\mathcal{L} x)(t, a) .
$$

In order that we are not observing an input or a zero boundary condition, we assume that rank $\left[\begin{array}{cc}M_{11} & M_{12} \\ M_{21} & M_{22} \\ C_{1} & C_{2}\end{array}\right]=$ $n+\operatorname{rank}\left[\begin{array}{ll}C_{1} & C_{2}\end{array}\right]$. In other words we choose the observations at the boundary to be complementary to the boundary control (2.8). Notice again that both the boundary input and output variables are defined as linear combinations of the co-energy variables $\mathcal{L} x$ (obtained from the variational derivative of the energy) restricted to the boundary, which appears naturally in the energy balance equation (2.5).

Equations (2.6)-(2.9) give a system with boundary control and observation. The aim of this paper is to study solutions of this system. For this we define the notion of well-posedness. Note that this is the adaptation 
of a more general notion to our class of p.d.e.'s, see [23] or [26]. By $H^{1}$ we denote the Sobolev space of square integrable functions, whose derivative is also square integrable.

Definition 2.1. Consider the system (2.6)-(2.9) and let $k$ be the dimension of $u$. This system is well-posed if there exists a $t_{f}>0$ and $m_{f}$ such that the following holds:

(1) The operator $A$ defined as $P_{0}+P_{1} \frac{\partial}{\partial z} \mathcal{L}$ with domain,

$$
D(A)=\left\{x_{0} \in X \mid \mathcal{L} x_{0} \in H^{1}\left((a, b) ; \mathbb{R}^{n}\right),\left[\begin{array}{ll}
M_{11} & M_{12} \\
M_{21} & M_{22}
\end{array}\right]\left[\begin{array}{l}
\left(\mathcal{L} x_{0}\right)(b) \\
\left(\mathcal{L} x_{0}\right)(a)
\end{array}\right]=0\right\}
$$

is the infinitesimal generator of a $C_{0}$-semigroup on $X$.

(2) The following inequality holds for all $\mathcal{L} x_{0} \in H^{1}\left((a, b) ; \mathbb{R}^{n}\right)$ and $u \in C^{2}\left(\left[0, t_{f}\right) ; \mathbb{R}^{k}\right)$ with $u(0)=$ $M_{21}\left(\mathcal{L} x_{0}\right)(b)+M_{22}\left(\mathcal{L} x_{0}\right)(a)$, and $0=M_{11}\left(\mathcal{L} x_{0}\right)(b)+M_{12}\left(\mathcal{L} x_{0}\right)(a)$

$$
\left\|x\left(t_{f}\right)\right\|_{\mathcal{L}}^{2}+\int_{0}^{t_{f}}\|y(t)\|^{2} \mathrm{~d} t \leq m_{f}\left[\left\|x_{0}\right\|_{\mathcal{L}}^{2}+\int_{0}^{t_{f}}\|u(t)\|^{2} \mathrm{~d} t\right] .
$$

Item (1) of this definition implies that the homogeneous p.d.e., i.e. (2.6), (2.7), and with (2.8) put to zero, has a unique (weak) solution for every initial condition in $X$. This implies that the inhomogeneous equation possesses a unique solution provided that the input and the initial condition are sufficiently smooth. This is formalized in the following lemma, see Malinen [19].

Lemma 2.2. Let $t_{f}$ be a positive real number. Assume further that condition (1) of Definition 2.1 is satisfied. Then for every $\mathcal{L} x_{0} \in H^{1}\left((a, b) ; \mathbb{R}^{n}\right)$ and every $u(\cdot) \in C^{2}\left(\left[0, t_{f}\right) ; \mathbb{R}^{k}\right)$ with $u(0)=M_{21}\left(\mathcal{L} x_{0}\right)(b)+M_{22}\left(\mathcal{L} x_{0}\right)(a)$, and $0=M_{11}\left(\mathcal{L} x_{0}\right)(b)+M_{12}\left(\mathcal{L} x_{0}\right)(a)$, there exists a unique classical solution of $(2.6)-(2.8)$ on $\left[0, t_{f}\right]$. Furthermore, the output (2.9) is well-defined and $y(\cdot)$ is continuous.

Since $\mathcal{L}^{-1} H^{1}\left((a, b) ; \mathbb{R}^{n}\right)$ and $C^{2}\left(\left[0, t_{f}\right) ; \mathbb{R}^{k}\right)$ are dense linear subspaces of $X$ and $L^{2}\left(\left[0, t_{f}\right) ; \mathbb{R}^{k}\right)$, respectively, we find that if $(2.10)$ holds for all $\mathcal{L} x_{0} \in H^{1}\left((a, b) ; \mathbb{R}^{n}\right)$ and $u \in C^{2}\left(\left[0, t_{f}\right), \mathbb{R}^{k}\right)$, then $(2.10)$ holds for all $x_{0} \in X$ and $u \in L^{2}\left(\left[0, t_{f}\right) ; \mathbb{R}^{k}\right)$. Hence if the system is well-posed, then (2.6)-(2.9) has for every initial condition and every square integrable input a unique (weak) solution, and (2.10) still holds.

For a well-posed system one can define the transfer function. Basically, this means that for an input of the form $u_{0} \exp (s t)$, one finds a state trajectory and an output of the form $y(t)=G(s) u_{0} \exp (s t)$. The function $G(s)$ is the transfer function, see Zwart [28]. For a well-posed system the transfer function exists and is bounded on some right-half plane.

Definition 2.3. Let $G(s)$ be the transfer function of (2.6)-(2.9). The system (2.6)-(2.9) is regular when $\lim _{s \rightarrow \infty} G(s)$ exists. If the system $(2.6)-(2.9)$ is regular, then the feed-through term $D$ is defined as $D=$ $\lim _{s \rightarrow \infty} G(s)$.

In the sequel we state the main result of this paper which gives the conditions under which, for the class of systems given in (2.6)-(2.9), the inequality (2.10) of the Definition 2.1 is necessarily satisfied if the condition (1) is satisfied. Furthermore, when the condition (1) holds, then the transfer function corresponding to the wellposed system is regular. We formulate this in the following theorem.

Theorem 2.4. Consider the partial differential equation (2.6)-(2.9) on the spatial interval $[a, b]$, with $x(t, z)$ taking values in $\mathbb{R}^{n}$. Let $X$ be the Hilbert space $L^{2}\left((a, b) ; \mathbb{R}^{n}\right)$ with inner product $(2.4)$. Furthermore, assume that

- $P_{1}$ is real-valued, invertible, and symmetric, i.e., $P_{1}^{T}=P_{1}$.

- $\mathcal{L}$ is a (real) multiplication operator on $L^{2}\left((a, b) ; \mathbb{R}^{n}\right)$. For every $z \in[a, b], \mathcal{L}(z)$ is symmetric and satisfies $0<m I \leq \mathcal{L}(z) \leq M I$, for some $m$ and $M$ independent of $z \in[a, b]$. 
- The multiplication operator $P_{1} \mathcal{L}$ can be written as

$$
P_{1} \mathcal{L}(z)=S^{-1}(z) \Delta(z) S(z)
$$

with $\Delta(z)$ a diagonal multiplication operator, and both $\Delta(z)$ and $S(z)$ are continuously differentiable.

- $\left[\begin{array}{ll}M_{11} & M_{12} \\ M_{21} & M_{22}\end{array}\right]$ is a $n \times 2 n$ matrix with rank $n$.

- $\operatorname{rank}\left[\begin{array}{cc}M_{11} & M_{12} \\ M_{21} & M_{22} \\ C_{1} & C_{2}\end{array}\right]=n+\operatorname{rank}\left[\begin{array}{ll}C_{1} & C_{2}\end{array}\right]$.

If the homogeneous p.d.e., i.e., $u \equiv 0$, generates a $C_{0}$-semigroup on $X$, then the system (2.6)-(2.9) is wellposed, and the corresponding transfer function $G$ is regular. Furthermore, we have that $\lim _{\operatorname{Re}(s) \rightarrow \infty} G(s)=$ $\lim _{s \rightarrow \infty} G(s)$.

As mentioned before, the last condition in the theorem tells us that we will not be observing inputs or boundary conditions which are set to zero. In other words, we are really observing a part of the state.

The third condition tells us that $P_{1} \mathcal{L}$ is diagonalizable via a continuously differentiable basis transformation. In Kato [11], Chapter II, one can find conditions on $P_{1} \mathcal{L}(z)$ such that this is possible. For simplicity, we have assumed that $\Delta(z)$ is continuously differentiable. However, the above theorem also holds if this does not hold.

Since $\mathcal{L}(z)>m I$, we see that for every $z \in[a, b]$ the signature of the matrix $\mathcal{L}(z)^{\frac{1}{2}} P_{1} \mathcal{L}(z)^{\frac{1}{2}}$ equals the signature of $P_{1}$. This implies that the signature of $\mathcal{L}(z)^{\frac{1}{2}} P_{1} \mathcal{L}(z)^{\frac{1}{2}}$ is independent of $z$. Furthermore, a simple calculation gives that the eigenvalues of $\mathcal{L}(z)^{\frac{1}{2}} P_{1} \mathcal{L}(z)^{\frac{1}{2}}$ are equal to the eigenvalues of $P_{1} \mathcal{L}(z)$. Concluding, we see that for all $z \in[a, b]$ zero is not an eigenvalue of $P_{1} \mathcal{L}(z)$, and that the number of negative and positive eigenvalues of $P_{1} \mathcal{L}(z)$ is independent of $z$. Thus without loss of generality, we may assume that

$$
\Delta(z)=\left(\begin{array}{cc}
\Lambda(z) & 0 \\
0 & \Theta(z)
\end{array}\right)
$$

where $\Lambda(z)$ is a diagonal (real) matrix, with positive functions on the diagonal, and $\Theta(z)$ is a diagonal (real) matrix, with negative functions on the diagonal.

In [15] necessary and sufficient conditions are given such that the homogeneous p.d.e. (2.6)-(2.8) generates a contraction semigroup and also gives the dissipation inequalities associated with the choices of the boundary output variables (2.9). As a consequence of the Theorem 2.4, these systems are furthermore proved to be well-posed and regular.

We prove this theorem in two main steps. First, in Section 3, we assume that $P_{1} \mathcal{L}$ is diagonal and prove the theorem in this case. Secondly, in Section 4, we consider the general case and use the fact that Theorem 2.4 remains valid under a basis transformation.

As a consequence of our proof we obtain a matrix condition for item 1 in Theorem 2.4, see Remark 4.2. This extends the matrix condition of [21], Theorem 3.1, to mixed boundary conditions.

\section{Proof of Theorem 2.4 For the diagonal CASE}

In this section, we prove Theorem 2.4 when $P_{1} \mathcal{L}$ is diagonal. For this we need the following two lemmas.

Lemma 3.1. Let $\lambda(z)$ be a positive continuously differentiable function on the interval $[a, b]$. With this function we define the scalar system

$$
\frac{\partial w}{\partial t}(t, z)=\frac{\partial}{\partial z}(\lambda(z) w(t, z)), \quad w(0, z)=w_{0}(z) \quad z \in[a, b]
$$

The value at $b$ we choose as input

$$
u(t)=\lambda(b) w(t, b)
$$


and as output we choose the value on the other end

$$
y(t)=\lambda(a) w(t, a)
$$

The system (3.1)-(3.3) is a well-posed system on the state space $L^{2}(a, b)$, and its solution is given as

$$
w(t, z)=f(p(z)+t) \lambda(z)^{-1}
$$

where

$$
\begin{aligned}
p(z) & =\int_{a}^{z} \lambda(\zeta)^{-1} \mathrm{~d} \zeta \\
f(p(z)) & =\lambda(z) w_{0}(z), \quad z \in[a, b] \\
f(p(b)+t) & =u(t), \quad t>0 .
\end{aligned}
$$

The transfer function is given by $G(s)=\mathrm{e}^{-p(b) s}$ which satisfies

$$
\lim _{\operatorname{Re}(s) \rightarrow \infty} G(s)=0 .
$$

Proof. It is easy to see that (3.4)-(3.7) is a classical solution of (3.1)-(3.2) provided $w_{0}$ is continuously differentiable and $\lambda(b) w_{0}(b)=u(0)$. Let $w(t, z)$ be this classical solution, then

$$
\begin{aligned}
\frac{\partial}{\partial t} \int_{a}^{b} w^{*}(t, z) \lambda(z) w(t, z) \mathrm{d} z & =\int_{a}^{b}\left[\frac{\partial}{\partial z}[\lambda(z) w(t, z)]^{*} \lambda(z) w(t, z)+w(t, z)^{*} \lambda(z) \frac{\partial}{\partial z}[\lambda(z) w(t, z)]\right] \mathrm{d} z \\
& =\left.[\lambda(z) w(t, z)]^{*} \lambda(z) w(t, z)\right|_{a} ^{b} \\
& =|u(t)|^{2}-|y(t)|^{2}
\end{aligned}
$$

Thus for all $t>0$ we have that

$$
\int_{a}^{b} w^{*}(t, z) \lambda(z) w(t, z) \mathrm{d} z-\int_{a}^{b} w^{*}(0, z) \lambda(z) w(0, z) \mathrm{d} z=\int_{0}^{t}|u(\tau)|^{2} \mathrm{~d} \tau-\int_{0}^{t}|y(\tau)|^{2} \mathrm{~d} \tau .
$$

Since $\lambda$ is strictly positive, we have that the norm $\int_{a}^{b} w^{*}(t, z) \lambda(z) w(t, z) \mathrm{d} z$ is equivalent to the $L^{2}(a, b)$-norm, and so on a dense set an inequality like (2.10) is satisfied. Thus the system is well-posed, see also the remark following Definition 2.1.

The transfer function $G(s)$ is constructed by finding for $s \in \mathbb{C}$ and for all $u_{0}$ a triple $\left(u_{s}(t), w_{s}(t, z), y(t)\right)=$ $\left(u_{0} \mathrm{e}^{s t}, w_{0}(z) \mathrm{e}^{s t}, y_{0} \mathrm{e}^{s t}\right)$ satisfying (3.1)-(3.3). If such a triple can be constructed and $y_{0}$ is uniquely depending on $u_{0}$, then $G(s)$ is defined as $G(s) u_{0}=y_{0}$, see [28]. Substituting a triple of this form in the p.d.e., gives

$$
s w_{0}(z)=\frac{\partial}{\partial z}\left(\lambda(z) w_{0}(z)\right), \quad u_{0}=\lambda(b) w_{0}(b), \quad y_{0}=\lambda(a) w_{0}(a)
$$

Thus $w_{0}(z)=u_{0} \lambda(z)^{-1} \exp (s(p(z)-p(b)))$, and $y_{0}=u_{0} \exp (-s p(b))$. This gives the expression for the transfer function. The property (3.8) follows directly from it and the fact that $p(b)>0$.

For a p.d.e. with negative coefficient, we obtain a similar result.

Lemma 3.2. Let $\theta(z)$ be a negative continuously differentiable function on the interval $[a, b]$. With this function we define the scalar system

$$
\frac{\partial w}{\partial t}(t, z)=\frac{\partial}{\partial z}(\theta(z) w(t, z)), \quad w(0, z)=w_{0}(z) \quad z \in[a, b] .
$$


The value at a we choose as input

$$
u(t)=\theta(a) w(t, a)
$$

and as output we choose the value on the other end

$$
y(t)=\theta(b) w(t, b)
$$

The system (3.10)-(3.12) is a well-posed system on the state space $L^{2}(a, b)$, and its solution is given as

$$
w(t, z)=f(n(z)+t) \theta(z)^{-1}
$$

where

$$
\begin{aligned}
n(z) & =\int_{a}^{z} \theta(\zeta)^{-1} \mathrm{~d} \zeta \\
f(n(z)) & =\theta(z) w_{0}(z), \quad z \in[a, b] \\
f(t) & =u(t), \quad t>0 .
\end{aligned}
$$

The transfer function is given by $G(s)=\mathrm{e}^{n(b) s}$ and satisfies (3.8).

We use these two lemmas to prove Theorem 2.4 when $P_{1} \mathcal{L}$ is diagonal and the input space has dimension $n$. Consider the following diagonal hyperbolic system on the spatial interval $z \in[a, b]$

$$
\frac{\partial}{\partial t}\left(\begin{array}{l}
x_{+}(t, z) \\
x_{-}(t, z)
\end{array}\right)=\frac{\partial}{\partial z}\left[\left(\begin{array}{cc}
\Lambda(z) & 0 \\
0 & \Theta(z)
\end{array}\right)\left(\begin{array}{l}
x_{+}(t, z) \\
x_{-}(t, z)
\end{array}\right)\right]
$$

where $\Lambda(z)$ is a diagonal (real) matrix, with positive functions on the diagonal, and $\Theta(z)$ is a diagonal (real) matrix, with negative functions on the diagonal. Furthermore, we assume that $\Lambda$ and $\Theta$ are continuously differentiable.

With this p.d.e. we associate the following boundary control and observation

$$
\begin{aligned}
& u_{s}(t):=\left(\begin{array}{c}
\Lambda(b) x_{+}(t, b) \\
\Theta(a) x_{-}(t, a)
\end{array}\right), \\
& y_{s}(t):=\left(\begin{array}{c}
\Lambda(a) x_{+}(t, a) \\
\Theta(b) x_{-}(t, b)
\end{array}\right) .
\end{aligned}
$$

Theorem 3.3. Consider the p.d.e. (3.17) with $u_{s}$ and $y_{s}$ as defined in (3.18) and (3.19), respectively.

- The system defined by (3.17)-(3.19) is well-posed and regular. Furthermore, its transfer function converges to zero for $\operatorname{Re}(s) \rightarrow \infty$.

- To the p.d.e. (3.17) we define a new set of inputs and outputs. The new input u(t) is written as

$$
u(t)=K u_{s}(t)+Q y_{s}(t)
$$

where $K$ and $Q$ are two square matrices, with $[K, Q]$ of rank $n$. The new output is written as

$$
y(t)=O_{1} u_{s}(t)+O_{2} y_{s}(t)
$$

where $\mathrm{O}_{1}$ and $\mathrm{O}_{2}$ are some matrices. For the system (3.17) with input $u(t)$ and output $y(t)$, we have the following possibilities:

(1) If $K$ is invertible, then the system (3.17)-(3.21) is well-posed and regular. Furthermore, its transfer function converges to $O_{1} K^{-1}$ for $\operatorname{Re}(s) \rightarrow \infty$. 


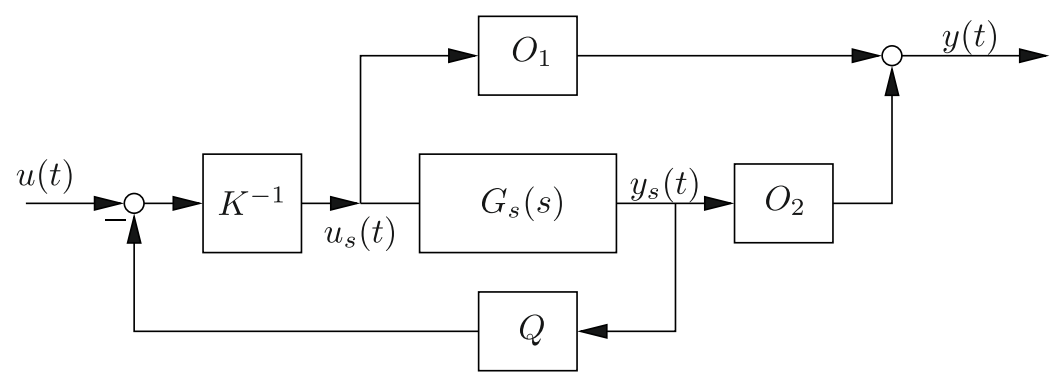

FiguRE 1. The system (3.17) with input (3.20) and output (3.21).

(2) If $K$ is not invertible, then the operator $A_{K}$ defined as

$$
A_{K}\left(\begin{array}{c}
g_{+}(z) \\
g_{-}(z)
\end{array}\right)=\frac{\partial}{\partial z}\left[\left(\begin{array}{cc}
\Lambda(z) & 0 \\
0 & \Theta(z)
\end{array}\right)\left(\begin{array}{c}
g_{+}(z) \\
g_{-}(z)
\end{array}\right)\right]
$$

with domain

$$
\begin{aligned}
D\left(A_{K}\right)= & \left\{\left(\begin{array}{c}
g_{+}(z) \\
g_{-}(z)
\end{array}\right) \in H^{1}\left((a, b), \mathbb{R}^{n}\right) \mid\right. \\
& \left.K\left(\begin{array}{c}
\Lambda(b) g_{+}(b) \\
\Theta(a) g_{-}(a)
\end{array}\right)+Q\left(\begin{array}{c}
\Lambda(a) g_{+}(a) \\
\Theta(b) g_{-}(b)
\end{array}\right)=0\right\}
\end{aligned}
$$

does not generate a $C_{0}$-semigroup on $L^{2}\left((a, b) ; \mathbb{R}^{n}\right)$.

Note that the last item implies that the homogeneous p.d.e. does not have a well-defined solution, when $K$ is not invertible.

Proof. The first item is a direct consequence of Lemmas 3.1 and 3.2 by noticing that the system (3.17)-(3.19) is built out of copies of the system (3.1)-(3.3) and the system (3.10)-(3.12). Furthermore, these sub-systems do not interact with each other.

For the proof of the first part of the second assertion, with $K$ invertible, we rewrite the new input, as $u_{s}(t)=K^{-1} u(t)-K^{-1} Q y_{s}(t)$. This can be seen as a feedback interconnection on the system (3.17)-(3.19), as is depicted in Figure 1. The system contains one feedback loop with gain matrix $K^{-1} Q$. By Weiss [26], see also Staffans [23], Chapter 7, we have that if $I+G_{s}(s) K^{-1} Q$ is invertible on some right-half plane and if this inverse is bounded on a right-half plane, then the closed loop system is well-posed. $\operatorname{Since} \lim _{\operatorname{Re}(s) \rightarrow \infty} G_{s}(s)=0$, we see that this holds for every $K^{-1}$ and $Q$. So under the assumption that $K$ is invertible, we find that (3.17) with input and output given by (3.20) and (3.21) is well-posed. The regularity follows easily. By regarding the loops in Figure 1 we see that the feed-though term is $O_{1} K^{-1}$.

So it remains to show that there is no $C_{0}$-semigroup when $K$ is non-invertible. Since $K$ is singular, there exists a non-zero $v \in \mathbb{R}^{n}$ such that $v^{T} K=0$. Since $[K, Q]$ has full rank, we know that $q^{T}:=v^{T} Q \neq 0$. So at least one of the components of $q$ is unequal to zero. For the sake of the argument, we assume that this holds for the first one.

If $A_{K}$ would be the infinitesimal generator of a $C_{0}$-semigroup, then for all $x_{0} \in D\left(A_{K}\right)$ the abstract differential equation

$$
\dot{x}(t)=A_{K} x(t), \quad x(0)=x_{0}
$$

would have classical solution, i.e., for all $t>0, x(t)$ is differentiable, it is an element of $D\left(A_{K}\right)$, and it satisfies (3.24). Hence by (3.23), we have that $x(t)$ is an element of $H^{1}$. Since we are working in a one dimensional spatial domain, we have that functions in $H^{1}$ are continuous. So we have that for every $t, x(t)$ is a continuous function of $z$ satisfying the boundary conditions in (3.23). 
So if $A_{K}$ would generate a $C_{0}$-semigroup, then for every $x_{0} \in D\left(A_{K}\right)$ there would be a function $x(t, z):=$ $\left(\begin{array}{l}x_{+}(t, z) \\ x_{-}(t, z)\end{array}\right)$ which is a (mild) solution to the p.d.e. (3.17), and satisfies for all $t>0$ the boundary condition

$$
K\left(\begin{array}{c}
\Lambda(b) x_{+}(t, b) \\
\Theta(a) x_{-}(t, a)
\end{array}\right)+Q\left(\begin{array}{c}
\Lambda(a) x_{+}(t, a) \\
\Theta(b) x_{-}(t, b)
\end{array}\right)=0
$$

Using the vectors $v$ and $q$, we see that this $x(t, z)$ must satisfy

$$
0=q^{T}\left(\begin{array}{c}
\Lambda(a) x_{+}(t, a) \\
\Theta(b) x_{-}(t, b)
\end{array}\right), \quad t>0
$$

Now we construct an initial condition in $D\left(A_{K}\right)$, for which this equality does not hold. Note that we have chosen the first component of $q$ unequal to zero.

The initial condition $x_{0}$ is chosen to have all components zero except for the first one. For this first component we choose an arbitrary function in $H^{1}(a, b)$ which is zero at $a$ and $b$, but nonzero everywhere on the open set $(a, b)$. It is clear that this initial condition is in the domain of $A_{K}$. Now we solve (3.17).

Standard p.d.e. theory gives that the solution of (3.17) can be written as, see also Lemmas 3.1 and 3.2 ,

$$
\begin{aligned}
x_{+, m}(t, z) & =f_{+, m}\left(p_{m}(z)+t\right) \lambda_{m}(z)^{-1}, \\
x_{-, l}(t, z) & =f_{-, l}\left(n_{l}(z)+t\right) \theta_{l}(z)^{-1},
\end{aligned}
$$

where $\lambda_{m}$ and $\theta_{l}$ are the $m$ th and the $l$ th diagonal element of $\Lambda$ and $\Theta$, respectively. Furthermore, $p_{m}(z)=$ $\int_{a}^{z} \lambda_{m}(\zeta)^{-1} \mathrm{~d} \zeta, n_{l}(z)=\int_{a}^{z} \theta_{l}(\zeta)^{-1} \mathrm{~d} \zeta$, see also Lemmas 3.1 and 3.2. The functions $f_{+}, f_{-}$need to be determined from the boundary and initial conditions.

Using the initial condition we have that $f_{+, m}\left(p_{m}(z)\right)=\lambda_{m}(z) x_{0,+, m}(z)$ and $f_{-, l}\left(n_{l}(z)\right)=\theta_{l}(z) x_{0,-, l}(z)$. Since $p_{m}>0$, and $n_{l}<0$, we see that the initial condition determines $f_{+}$on a (small) positive interval, and $f_{-}$ on a small negative interval. By our choice of the initial condition, we find that

$$
\begin{aligned}
& f_{+, 1}(\xi)=\lambda_{1}(\xi) x_{0,+, 1}(\xi) \\
& f_{+, m}(\xi)=0 \\
& \xi \in\left[0, p_{1}(b)\right), \\
& f_{-, l}(\xi)=0 \\
& \xi \in\left[0, p_{m}(b)\right), \quad m \geq 2, \\
& \xi \in\left[n_{l}(b), 0\right), \quad l \geq 1 .
\end{aligned}
$$

The solution $x(t, z)$ must also satisfy (3.25), thus for all $t>0$ we have that

$$
0=q^{T}\left(\begin{array}{c}
f_{+}(t) \\
f_{-}(n(b)+t)
\end{array}\right)
$$

Combining this with (3.26), we find

$$
0=q_{1} f_{+, 1}\left(p_{1}(z)\right)=q_{1} x_{0,+, 1}(z) \lambda_{1}^{-1}(z)
$$

on some interval $[a, \beta]$. Since $q_{1}$ and $\lambda_{1}$ are unequal to zero, we find that $x_{0}$ must be zero on some interval. This is in contradiction with our choice of the initial condition. Thus $A_{K}$ cannot be the infinitesimal generator of a $C_{0}$-semigroup.

\section{Proof of Theorem 2.4, General Case}

In this section we use the results of the previous section to prove Theorem 2.4. We begin with a useful lemma. Its proof is based on the feedback results of Staffans [23], Chapter 7. 
Lemma 4.1. The system (2.6)-(2.9) is well-posed if and only if the system

$$
\frac{\partial x}{\partial t}(t, x)=P_{1} \frac{\partial}{\partial z}(\mathcal{L} x)(t, z)
$$

with inputs, outputs given by (2.7)-(2.9) is well-posed.

Let $G(s)$ denote the transfer function of (2.6)-(2.9) and $G_{0}(s)$ the transfer function of (4.1) with (2.7)-(2.9). Then

and

$$
\lim _{s \rightarrow \infty} G(s)=\lim _{s \rightarrow \infty} G_{0}(s),
$$

$$
\lim _{\operatorname{Re}(s) \rightarrow \infty} G(s)=\lim _{\operatorname{Re}(s) \rightarrow \infty} G_{0}(s) .
$$

Proof. We consider the system (2.6)-(2.10) with (2.6) replaced by

$$
\frac{\partial x}{\partial t}(t, z)=P_{1} \frac{\partial}{\partial z}(\mathcal{L} x)(t, z)+u_{1}(t, z), \quad x(0, z)=x_{0}(z)
$$

and the additional observation

$$
y_{1}(t, z)=x(t, z) \text {. }
$$

Since the extra input and output operator is a bounded operator, it is easy to show that this system is wellposed when (4.1) with inputs, outputs given by (2.7)-(2.9) is well-posed, see also [24], p. 164. Furthermore, the feed-through from $\left(u, u_{1}\right)$ to $y_{1}$ is zero. Using [23], Chapter 7 , we see that the feedback $u_{1}=P_{0} u_{1}$ is allowed, and gives a well-posed system. This closed loop system is clearly equal to (2.6)-(2.10). The revised direction is proved similarly by adding an extra input and output to the system (2.6)-(2.10).

This lemma tells us that we may ignore any bounded linear term involving $x$.

By the third assumption of Theorem 2.4, the matrices $P_{1}$ and $\mathcal{L}$ satisfy equation $(2.11)$ :

$$
P_{1} \mathcal{L}(z)=S^{-1}(z) \Delta(z) S(z)
$$

With this we introduce the new state vector

$$
\tilde{x}(t, z)=S(z) x(t, z), \quad z \in[a, b]
$$

Under this basis transformation, the p.d.e. (2.6) becomes

$$
\begin{gathered}
\frac{\partial \tilde{x}}{\partial t}(t, z)=\frac{\partial}{\partial z}(\Delta \tilde{x})(t, z)+S(z) \frac{\mathrm{d} S^{-1}(z)}{\mathrm{d} z} \Delta(z) \tilde{x}(t, z)+S(z) P_{0}(z) S(z)^{-1} \tilde{x}(t, z) \\
x(0, z)=S(z) x_{0}(z)=\tilde{x}_{0}(z) .
\end{gathered}
$$

The relations (2.7)-(2.9) become

$$
\begin{aligned}
0 & =M_{11} P_{1}^{-1} S^{-1}(b) \Delta(b) \tilde{x}(t, b)+M_{12} P_{1}^{-1} S^{-1}(a) \Delta(a) \tilde{x}(t, a) \\
& =\tilde{M}_{11} \Delta(b) \tilde{x}(t, b)+\tilde{M}_{12} \Delta(a) \tilde{x}(t, a) \\
u(t) & =M_{21} P_{1}^{-1} S^{-1}(b) \Delta(b) \tilde{x}(t, b)+M_{22} P_{1}^{-1} S^{-1}(a) \Delta(a) \tilde{x}(t, a) \\
& =\tilde{M}_{21} \Delta(b) \tilde{x}(t, b)+\tilde{M}_{22} \Delta(a) \tilde{x}(t, a) \\
y(t) & =C_{1} P_{1}^{-1} S^{-1}(b) \Delta(b) \tilde{x}(t, b)+C_{2} P_{1}^{-1} S^{-1}(a) \Delta(a) \tilde{x}(t, a) \\
& =\tilde{C}_{1} \Delta(b) \tilde{x}(t, b)+\tilde{C}_{2} \Delta(a) \tilde{x}(t, a) .
\end{aligned}
$$


We introduce $\tilde{M}=\left(\begin{array}{cc}\tilde{M}_{11} & \tilde{M}_{12} \\ \tilde{M}_{21} & \tilde{M}_{22}\end{array}\right)$ with

$$
\left(\begin{array}{cc}
\tilde{M}_{j 1} & \tilde{M}_{j 2}
\end{array}\right)=\left(\begin{array}{cc}
M_{j 1} & M_{j 2}
\end{array}\right)\left(\begin{array}{cc}
P_{1}^{-1} S(b)^{-1} & 0 \\
0 & P_{1}^{-1} S(a)^{-1}
\end{array}\right), \quad j=1,2,
$$

and

$$
\tilde{C}=\left(\begin{array}{cc}
\tilde{C}_{1} & \tilde{C}_{2}
\end{array}\right)=\left(\begin{array}{ll}
C_{1} & C_{2}
\end{array}\right)\left(\begin{array}{cc}
P_{1}^{-1} S(b)^{-1} & 0 \\
0 & P_{1}^{-1} S(a)^{-1}
\end{array}\right) .
$$

Since the matrix $\left(\begin{array}{cc}P_{1}^{-1} S(b)^{-1} & 0 \\ 0 & P_{1}^{-1} S(a)^{-1}\end{array}\right)$ has full rank, we see that the rank conditions in Theorem 2.4 imply similar rank conditions for $\tilde{M}$ and $\tilde{C}$.

Using Lemma 4.1 we see that we only have to prove the result for the p.d.e.

$$
\frac{\partial \tilde{x}}{\partial t}(t, z)=\frac{\partial}{\partial z}(\Delta \tilde{x})(t, z)
$$

with boundary conditions, inputs, and outputs as described in (4.8)-(4.10).

It is clear that if condition (4.8) is not present, then Theorem 3.3 gives that the above system is well-posed and regular if and only if the homogeneous p.d.e. generates a $C_{0}$-semigroup on $L^{2}\left((a, b) ; \mathbb{R}^{n}\right)$. Since the state transformation (4.6) defines a bounded mapping on $L^{2}\left((a, b) ; \mathbb{R}^{n}\right)$, we have proved Theorem 2.4 provided there is no condition (2.7).

Thus it remains to prove Theorem 2.4 if we have put part of the boundary conditions to zero. Or equivalently, to prove that the system (4.8)-(4.11) is well-posed and regular if and only if the homogeneous p.d.e. generates a $C_{0}$-semigroup.

We replace (4.8) by

$$
v(t)=\tilde{M}_{11} \Delta(b) \tilde{x}(t, b)+\tilde{M}_{12} \Delta(a) \tilde{x}(t, a),
$$

where we regard $v$ as a new input. We consider the system consisting of the p.d.e. (4.11) with the new extended input

$$
\left(\begin{array}{c}
v(t) \\
u(t)
\end{array}\right)=\left(\begin{array}{c}
\tilde{M}_{11} \\
\tilde{M}_{21}
\end{array}\right) \Delta(b) \tilde{x}(t, b)+\left(\begin{array}{c}
\tilde{M}_{12} \\
\tilde{M}_{22}
\end{array}\right) \Delta(a) \tilde{x}(t, a)
$$

and the output (4.10). By doing so we have obtained a system without a condition (4.8). For this system we know that it is well-posed and regular if and only if the homogeneous equation generates a $C_{0}$-semigroup.

Assume that the system (4.11), (4.13) and (4.10) is well-posed, then we may choose any (locally) square input. In particular, since $u$ and $v$ are independent inputs, we may choose $v \equiv 0$. Thus the system (4.7)-(4.11) is well-posed and regular as well.

Assume next that the p.d.e. (4.11) with the extended input in (4.13) set to zero, does not generate a $C_{0}$-semigroup. Since this gives the same homogeneous p.d.e. as (4.11) with (4.8) and $u$ in (4.9) set to zero, we know that this p.d.e. does not generate a $C_{0}$-semigroup. This finally proves Theorem 2.4.

Concerning first item in Theorem 2.4, we have the following remarks.

\section{Remark 4.2.}

(1) From the proof of Theorem 2.4, we see that we obtain an equivalent matrix condition for condition 1 , i.e., first item of Theorem 2.4 holds if and only if $K$ is invertible, see Theorem 3.3. Since the matrix $K$ is obtained after a basis transformation, and depends on the negative and positive eigenvalues of $P_{1} \mathcal{L}$, it is not easy to rewrite this condition in a condition for $M_{i j}$.

(2) A semigroup can be extended to a group if the homogeneous p.d.e. has for every initial condition a solution for negative and positive time. Using once more the proof of Theorem 3.3, we see that $A$ in first item of Theorem 2.4 generates a group if and only if $K$ and $Q$ are invertible matrices. For non-mixed boundary conditions, this can be found in [21], Theorem 3.1. 


\section{EXAMPle OF THE VIBRATING STRING}

Consider again the example of the vibrating string written as the system of conservation laws (2.2).

This system belongs to the class of systems defined in the equation (2.3) with zero source term $\left(i . e . P_{0}=0\right)$ and

$$
P_{1}=\left(\begin{array}{cc}
0 & 1 \\
1 & 0
\end{array}\right), \quad \mathcal{L}=\left(\begin{array}{cc}
\frac{1}{\rho} & 0 \\
0 & T
\end{array}\right) .
$$

The operator $P_{1} \mathcal{L}$ of this system is diagonalizable:

$$
P_{1} \mathcal{L}=S(z)^{-1} \Delta(z) S(z)=\left(\begin{array}{cc}
\gamma & -\gamma \\
\frac{1}{\rho} & \frac{1}{\rho}
\end{array}\right)\left(\begin{array}{cc}
\gamma & 0 \\
0 & -\gamma
\end{array}\right)\left(\begin{array}{cc}
\frac{1}{2 \gamma} & \frac{\rho}{2} \\
\frac{-1}{2 \gamma} & \frac{\rho}{2}
\end{array}\right)
$$

where $\gamma^{2}=c=\frac{T}{\rho}$. We take $\gamma$ to be positive.

Hence the state transformation under which the p.d.e. becomes diagonal is

$$
\tilde{x}=\frac{1}{2}\left(\begin{array}{cc}
\frac{1}{\gamma} & \rho \\
\frac{-1}{\gamma} & \rho
\end{array}\right) x \text {. }
$$

Since we assumed that $\gamma>0$, we see that $\tilde{x}_{1}, \tilde{x}_{2}$ correspond to $x_{+}$, and $x_{-}$in equation (3.17), respectively and $\Lambda, \Theta$ to $\gamma$ and $-\gamma$, respectively. Hence we have that the input and output $u_{s}$ and $y_{s}$ defined for the diagonal system (3.17) by the equations (3.18)-(3.19) are expressed in the original coordinates by

$$
\begin{aligned}
& u_{s}(t)=\frac{1}{2}\left(\begin{array}{c}
x_{1}(t, b)+\gamma \rho x_{2}(t, b) \\
x_{1}(t, a)-\gamma \rho x_{2}(t, a)
\end{array}\right), \\
& y_{s}(t)=\frac{1}{2}\left(\begin{array}{c}
x_{1}(t, a)+\gamma \rho x_{2}(t, a) \\
x_{1}(t, b)-\gamma \rho x_{2}(t, b)
\end{array}\right) .
\end{aligned}
$$

This pair of boundary input and output variables consist in complementary linear combinations of the momentum $x_{1}$ and the the strain $x_{2}$ at the boundaries: however they lack an obvious physical interpretation. One could consider another choice of boundary input and outputs, for instance the velocity and the strain at the boundary points and choose as input $u_{1}(t)=\left(\begin{array}{c}\frac{x_{1}}{\rho}(t, b) \\ x_{2}(t, a)\end{array}\right)$ and as output $y_{1}(t)=\left(\begin{array}{c}\frac{x_{1}}{\rho}(t, a) \\ x_{2}(t, b)\end{array}\right)$. We may apply Theorem 3.3 to check whether this system is well-posed, and to find the feed-through. Expressing the input-output pair $\left(u_{1}, y_{1}\right)$ in $\left(u_{s}, y_{s}\right)$ gives

$$
\begin{aligned}
& u_{1}(t)=\left(\begin{array}{cc}
\frac{1}{\rho} & 0 \\
0 & \frac{-1}{\sqrt{T \rho}}
\end{array}\right) u_{s}(t)+\left(\begin{array}{cc}
0 & \frac{1}{\rho} \\
\frac{1}{\sqrt{T \rho}} & 0
\end{array}\right) y_{s}(t), \\
& y_{1}(t)=\left(\begin{array}{cc}
0 & \frac{1}{\rho} \\
\frac{1}{\sqrt{T \rho}} & 0
\end{array}\right) u_{s}(t)+\left(\begin{array}{cc}
\frac{1}{\rho} & 0 \\
0 & \frac{-1}{\sqrt{T \rho}}
\end{array}\right) y_{s}(t) .
\end{aligned}
$$

Hence

$$
\begin{array}{cc}
K=\left(\begin{array}{cc}
\frac{1}{\rho} & 0 \\
0 & \frac{-1}{\sqrt{T \rho}}
\end{array}\right) \quad Q=\left(\begin{array}{cc}
0 & \frac{1}{\rho} \\
\frac{1}{\sqrt{T \rho}} & 0
\end{array}\right), \\
O_{1}=\left(\begin{array}{cc}
0 & \frac{1}{\rho} \\
\frac{1}{\sqrt{T \rho}} & 0
\end{array}\right), & O_{2}=\left(\begin{array}{cc}
\frac{1}{\rho} & 0 \\
0 & \frac{-1}{\sqrt{T \rho}}
\end{array}\right) .
\end{array}
$$

Since $K$ is invertible, the system with the input-output pair $\left(u_{1}, y_{1}\right)$ is well-posed and regular, and the feedthrough term is given by $O_{1} K^{-1}=\left(\begin{array}{cc}0 & -\gamma \\ \frac{1}{\gamma} & 0\end{array}\right)$. 


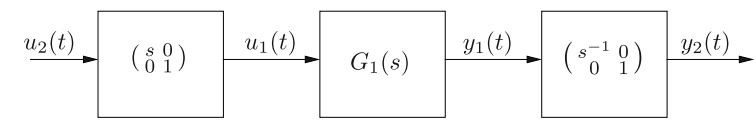

Figure 2. The system (2.1) with input and output (5.8) and (5.9).

Since $Q$ is also invertible, we have that the associated semigroup can be extended to a group.

Let us now interpret the boundary inputs $u_{1}$ and outputs $y_{1}$ in terms of the position $w$ in the second order p.d.e. (2.1) for which the change of coordinates with respect to the state variable of the system of conservation laws is $x_{1}=\rho \frac{\partial w}{\partial t}$ and $x_{2}=\frac{\partial w}{\partial z}$. One obtains the following expression of the boundary inputs and outputs: $u_{1}(t)=\left(\begin{array}{c}\frac{\partial w}{\partial t}(t, b) \\ \frac{\partial w}{\partial z}(t, a)\end{array}\right)$ and $y_{1}(t)=\left(\begin{array}{c}\frac{\partial w}{\partial t}(t, a) \\ \frac{\partial w}{\partial z}(t, b)\end{array}\right)$, respectively. However considering the p.d.e. (2.1), one could consider as one of the boundary inputs or output the position and not the velocity at the boundary points. This leads to consider:

$$
\begin{aligned}
& u_{2}(t)=\left(\begin{array}{c}
w(t, b) \\
\frac{\partial w}{\partial z}(t, a)
\end{array}\right) \\
& y_{2}(t)=\left(\begin{array}{c}
w(t, a) \\
\frac{\partial w}{\partial z}(t, b)
\end{array}\right) .
\end{aligned}
$$

These inputs and outputs a related to the previously defined input $u_{1}$ and output $y_{1}$ by integration and derivation with respect to time and this can be de-pictured as shown in Figure 2.

From this it is clear that the transfer function, $G_{2}(s)$ of the system with input $u_{2}$ and output $y_{2}$ is given by

$$
G_{2}(s)=\left(\begin{array}{cc}
s^{-1} & 0 \\
0 & 1
\end{array}\right) G_{1}(s)\left(\begin{array}{cc}
s & 0 \\
0 & 1
\end{array}\right) .
$$

Since for large real $s$ the transfer function $G_{1}(s)$ is approximately equal to $\left(\begin{array}{cc}0 & -\gamma \\ \gamma^{-1} & 0\end{array}\right)$, we see that $G_{2}(s)$ grows like $s$ for large $s$. Any well-posed system has a transfer function which is bounded in some right-half plane. Thus the system (2.1) with input (5.8) and output (5.9) is not well-posed.

Putting the input $u_{2}$ to zero, is the same as putting the input $u_{1}$ to zero. Hence the homogeneous p.d.e. will have a unique solution. Furthermore, for any initial condition the output corresponding to the homogeneous p.d.e. will stay square integrable. Hence the output is admissible, see Weiss [26]. On the other hand, since the transfer function grows to infinity for $s \rightarrow \infty$ the input will not be admissible.

Note that $u_{3}(t)=\frac{\partial w}{\partial z}(t, a), w(t, b)=0$, and $y_{3}(t)=y_{2}(t)$ will give a well-posed system.

\section{A SEMigroup EXAMPLE}

In this section, we show that it is possible to construct a closed operator $A$ and a coercive operator $\mathcal{L}$ such that $A$ with domain $D(A)$ is not the infinitesimal generator of a $C_{0}$-semigroup on the Hilbert space $X$, but the operator $A_{\mathcal{L}}$ defined as $A_{\mathcal{L}}:=A \mathcal{L}$ with domain all $x \in X$ such that $\mathcal{L} x \in D(A)$ does generate a $C_{0}$-semigroup on $X$. The example is based on the results of the previous section.

We begin with defining $A, \mathcal{L}$, and the state space. As state space we choose $L^{2}\left((0,1) ; \mathbb{R}^{2}\right)$. The coercive operator $\mathcal{L}$ is just

where $\gamma>0$. The operator $A$ is defined as

$$
\mathcal{L}=\left(\begin{array}{cc}
1 & 0 \\
0 & \gamma^{2}
\end{array}\right)
$$

$$
A\left(\begin{array}{l}
x_{1} \\
x_{2}
\end{array}\right)=\frac{\partial}{\partial z}\left[\left(\begin{array}{ll}
0 & 1 \\
1 & 0
\end{array}\right)\left(\begin{array}{l}
x_{1} \\
x_{2}
\end{array}\right)\right]
$$


with domain

$$
D(A)=\left\{x \in H^{1}\left((0,1) ; \mathbb{R}^{2}\right) \mid x_{1}(0)+x_{2}(0)=0, x_{2}(1)=0\right\} .
$$

Consider the p.d.e. associated to $A_{\mathcal{L}}=A \mathcal{L}$

$$
\frac{\partial}{\partial t}\left(\begin{array}{l}
x_{1} \\
x_{2}
\end{array}\right)(t, z)=\frac{\partial}{\partial z}\left[\left(\begin{array}{cc}
0 & 1 \\
1 & 0
\end{array}\right)\left(\begin{array}{cc}
1 & 0 \\
0 & \gamma^{2}
\end{array}\right)\left(\begin{array}{l}
x_{1} \\
x_{2}
\end{array}\right)(t, z)\right]
$$

with boundary conditions

$$
\left(\begin{array}{l}
0 \\
0
\end{array}\right)=\left(\begin{array}{ll}
1 & 1 \\
0 & 0
\end{array}\right)\left(\begin{array}{cc}
1 & 0 \\
0 & \gamma^{2}
\end{array}\right)\left(\begin{array}{l}
x_{1} \\
x_{2}
\end{array}\right)(t, 0)+\left(\begin{array}{ll}
0 & 0 \\
0 & 1
\end{array}\right)\left(\begin{array}{cc}
1 & 0 \\
0 & \gamma^{2}
\end{array}\right)\left(\begin{array}{l}
x_{1} \\
x_{2}
\end{array}\right)(t, 1) .
$$

So we have the p.d.e. (2.2) with $\rho=1$ and $T=\gamma^{2}$. We write the boundary condition (6.5) in terms of $u_{s}$ and $y_{s}$, see (5.2) and (5.3). This gives

$$
\left(\begin{array}{l}
0 \\
0
\end{array}\right)=\left(\begin{array}{cc}
0 & 1-\gamma \\
\gamma & 0
\end{array}\right) u_{s}(t)+\left(\begin{array}{cc}
1+\gamma & 0 \\
0 & -\gamma
\end{array}\right) y_{s}(t)
$$

From Theorem 3.3 we know that for $\gamma=1$ this does not generate a $C_{0}$-semigroup, and this is the only positive $\gamma$ with this property. Hence we have that $A$ does not generate a $C_{0}$-semigroup, whereas $A \mathcal{L}$ with $\gamma \in(0,1) \cup(1, \infty)$ does. Note that for every $\gamma>0$ the operator $\mathcal{L}^{-1} A \mathcal{L}$ with domain equal to the domain of $A_{\mathcal{L}}$ does not generate a $C_{0}$-semigroup. This follows from the fact that $\mathcal{L}^{-1} A \mathcal{L}$ is similar to $A$, see Exercise 2.5 of [2].

Finally, we remark, that it is easy to show that if $A$ generates a contraction semigroup on a Hilbert space, then $A \mathcal{L}$ is the infinitesimal generator of a $C_{0}$-semigroup.

\section{WELL-POSEDNESS ON $L^{q}$-SPACES}

The theorem as formulated in Section 2 can also be formulated in $L^{q}$ spaces.

Theorem 7.1. Let $1 \leq q<\infty$. Under the conditions of Theorem 2.4 we have that the homogeneous p.d.e. generates a $C_{0}$-semigroup on $L^{q}(a, b)$ if and only if the system is well-posed for $L^{q}$ inputs and outputs, that is

$$
\int_{0}^{t_{f}}\|y(t)\|^{q} \mathrm{~d} t+\int_{a}^{b}\left\|x\left(t_{f}, z\right)\right\|^{q} \mathrm{~d} z \leq m_{f}\left[\int_{0}^{t_{f}}\|u(t)\|^{q} \mathrm{~d} t+\int_{a}^{b}\left\|x_{0}(z)\right\|^{q} \mathrm{~d} z\right] .
$$

For the proof, we first prove the corresponding relation for the diagonal system, then we use a result of Staffans [23], Chapter 7, to show that the feedback is allowed if $K$ is invertible. Looking in the proof of Theorem 3.3, we see that in showing that $A_{K}$ is not a generator when $K$ is singular, we used only smooth functions. These functions are in $L^{q}$ for all $q$ and so this part of the proof easily carries over. The only essential part in the proof is to show Lemma 3.1, in particular equation (3.9), for $L^{q}$-functions.

Consider the p.d.e. (3.1)-(3.3). Its solution is given by (3.4)-(3.7). Now define $Q(t)$ as

$$
Q(t)=\int_{a}^{b}|w(t, z)|^{q} \lambda^{q-1}(z) \mathrm{d} z .
$$

Using (3.4) we see that $Q(t)$ is equal to

$$
Q(t)=\int_{a}^{b}|f(p(z)+t)|^{q} \lambda^{-1}(z) \mathrm{d} z .
$$


Performing the change of variables $\xi=p(z)+t$ in this integral and using that $\dot{p}=\lambda^{-1}$, see (3.5), we find that

$$
Q(t)=\int_{p(a)+t}^{p(b)+t}|f(\xi)|^{q} \mathrm{~d} \xi
$$

So we find that

$$
\begin{aligned}
Q\left(t_{f}\right)-Q(0) & =\int_{0}^{t_{f}} \dot{Q}(\tau) \mathrm{d} \tau \\
& =\int_{0}^{t_{f}}\left[|f(p(b)+\tau)|^{q}-|f(p(a)+\tau)|^{q}\right] \mathrm{d} \tau \\
& =\int_{0}^{t_{f}}|u(\tau)|^{q} \mathrm{~d} \tau-\int_{0}^{t_{f}}|y(\tau)|^{q} \mathrm{~d} \tau
\end{aligned}
$$

where we have used (3.3) and (3.7). Combining (7.2) and (7.4), gives he desired estimate.

We conclude this session by remarking that the equality (7.4) does not hold for $q=\infty$, i.e., for the sup norm. Simply take $f(\xi)=\xi$ and $\lambda=1$.

\section{Conclusion}

In this paper, we use physical port Hamiltonian modeling to study a class of hyperbolic partial differential equations on a one dimensional spatial domain with control and observation at the boundary. Even if for this class of systems the input mapping operator is unbounded, we show that the system is well-posed if and only if the state operator generates a $C_{0}$-semigroup. Furthermore, in this case the associated transfer function is regular and it is possible to give the expression of its direct transmission term. To prove this result we consider a basis state transformation to represent the system as a coupled set of delay lines. We regard this coupling as a feedback interconnection. The well posedness of the global system is given checking a condition on this feedback according to a result proposed by Weiss [26]. Furthermore, from the input and output definition in the diagonal case we characterize all the possible choices of boundary input and output that permit to obtain a well posed system and give, from his parametrization, the associated direct transmission term. With this work we showed that appropriate physical modeling is of great interest to prove properties of solutions associated to boundary control systems. Finally, we can remark that the class of systems treated in this paper is large enough to deal with well known examples from the literature as coupled wave equation (see [16], Chap. 6, and [3]), Timoshenko beam equation, convection equation. Currently, we try to generalize these results to systems with a dissipation term.

\section{REFERENCES}

[1] A. Cheng and K. Morris, Well-posedness of boundary control systems. SIAM J. Control Optim. 42 (2003) 1244-1265.

[2] R.F. Curtain and H.J. Zwart, An Introduction to Infinite-Dimensional Linear Systems Theory. Springer-Verlag, New York, USA (1995).

[3] R. Dáger and E. Zuazua, Wave Propagation, Observation and Control in 1-d Flexible Multi-structures, Matématiques 83 Applications 50. Springer-Verlag (2006).

[4] K.-J. Engel, M. Kramar Fijavž, R. Nagel, E. Sikolya, Vertex control of flows in networks. Netw. Heterog. Media 3 (2008) 709-722.

[5] B.-Z. Guo and Z.-C. Shao, Regularity of a Schödinger equation with Dirichlet control and collocated observation. Syst. Contr. Lett. 54 (2005) 1135-1142.

[6] B.-Z. Guo and Z.-C. Shao, Regularity of an Euler-Bernoulli equation with Neumann control and collocated observation. J. Dyn. Contr. Syst. 12 (2006) 405-418.

[7] B.-Z. Guo and Z.-X. Zhang, The regularity of the wave equation with partial Dirichlet control and collocated observation. SIAM J. Control Optim. 44 (2005) 1598-1613. 
[8] B.-Z. Guo and Z.-X. Zhang, Well-posedness and regularity for an Euler-Bernoulli plate with variable coefficients and boundary control and observation. MCSS 19 (2007) 337-360.

[9] B.-Z. Guo and Z.-X. Zhang, On the well-posedness and regularity of the wave equation with variable coefficients. ESAIM: COCV 13 (2007) 776-792.

[10] B.-Z. Guo and Z.-X. Zhang, Well-posedness of systems of linear elasticity with Dirichlet boundary control and observation. SIAM J. Control Optim. 48 (2009) 2139-2167.

[11] T. Kato, Perturbation Theory for Linear Operators. Corrected printing of the second edition, Springer-Verlag, Berlin, Germany (1980).

[12] I. Lasiecka and R. Triggiani, Control Theory for Partial Differential Equations I, Encyclopedia of Mathematics and its Applications 74. Cambridge University Press (2000).

[13] I. Lasiecka and R. Triggiani, Control Theory for Partial Differential Equations II, Encyclopedia of Mathematics and its Applications 75. Cambridge University Press (2000).

[14] Y. Le Gorrec, B.M. Maschke, H. Zwart and J.A. Villegas, Dissipative boundary control systems with application to distributed parameters reactors, in Proc. IEEE International Conference on Control Applications, Munich, Germany, October 4-6 (2006) 668-673.

[15] Y. Le Gorrec, H. Zwart and B. Maschke, Dirac structures and boundary control systems associated with skewsymmetric differential operators. SIAM J. Control Optim. 44 (2005) 1864-1892. A more detailed version is available at www.math.utwente.nl/publications, Memorandum No. 1730 (2004).

[16] Z.-H. Luo, B.-Z. Guo and O. Morgul, Stability and Stabilization of Infinite-Dimensional Systems with Applications. SpringerVerlag (1999).

[17] A. Macchelli and C. Melchiorri, Modeling and control of the Timoshenko beam, The distributed port Hamiltonian approach. SIAM J. Control Optim. 43 (2004) 743-767.

[18] B. Maschke and A.J. van der Schaft,Compositional modelling of distributed-parameter systems, in Advanced Topics in Control Systems Theory - Lecture Notes from FAP 2004, Lecture Notes in Control and Information Sciences, F. Lamnabhi-Lagarrigue, A. Loría and E. Panteley Eds., Springer (2005) 115-154.

[19] J. Malinen, Conservatively of time-flow invertible and boundary control systems. Research Report A479, Institute of Mathematics, Helsinki University of Technology, Finland (2004). See also Proceedings of the Joint 44th IEEE Conference on Decision and Control and European Control Conference (CDC-ECC'05).

[20] R.S. Phillips, Dissipative operators and hyperbolic systems of partial differential equations. Trans. Amer. Math. Soc. 90 (1959) 193-254.

[21] D.L. Russell, Controllability and stabilizability theory for linear partial differential equations: recent progress and open questions. SIAM Review 20 (1978) 639-739.

[22] A.J. van der Schaft and B.M. Maschke, Hamiltonian formulation of distributed parameter systems with boundary energy flow. J. Geometry Physics 42 (2002) 166-174.

[23] O. Staffans, Well-posed Linear Systems, Encyclopedia of Mathematics and its Applications 103. Cambridge University Press (2005).

[24] M. Tucsnak and G. Weiss, Observation and Control for Operator Semigroups, Birkhäuser Advanced Texts. Basler Lehrbücher (2009).

[25] J.A. Villegas, A Port-Hamiltonian Approach to Distributed Parameter Systems. Ph.D. Thesis, University of Twente, The Netherlands (2007). Available at http://doc.utwente.nl.

[26] G. Weiss, Regular linear systems with feedback. Math. Control Signals Syst. 7 (1994) 23-57.

[27] G. Weiss and R.F. Curtain, Exponential stabilization of a Rayleigh beam using collocated control. IEEE Trans. Automat. Contr. 53 (2008) 643-654.

[28] H. Zwart, Transfer functions for infinite-dimensional systems. Syst. Contr. Lett. 52 (2004) 247-255.

[29] H. Zwart, Y. Le Gorrec, B.M.J. Maschke and J.A. Villegas, Well-posedness and regularity for a class of hyperbolic boundary control systems, in Proceedings of the 17th International Symposium on Mathematical Theory of Networks and Systems, Kyoto, Japan (2006) 1379-1883. 